\title{
Identification of candidate target genes for human peripheral arterial disease using weighted gene co-expression network analysis
}

\author{
DE-XIN YIN ${ }^{1}$, HAO-MIN ZHAO ${ }^{1}$, DA-JUN SUN ${ }^{1}$, JIAN YAO $^{2}$ and DA-YONG DING ${ }^{3}$ \\ ${ }^{1}$ Department of Vascular Surgery, China-Japan Union Hospital of Jilin University, Changchun, Jilin 130033; \\ ${ }^{2}$ Department of Thoracic Surgery, Jilin Hospital of Jilin Province People's Hospital, Changchun, Jilin 130031; \\ ${ }^{3}$ Department of Gastroenterology Surgery, China-Japan Union Hospital of Jilin University, \\ Changchun, Jilin 130033, P.R. China
}

Received December 23, 2014; Accepted September 16, 2015

DOI: $10.3892 / \mathrm{mmr} .2015 .4450$

\begin{abstract}
The aim of the present study was to identify the potential treatment targets of peripheral arterial disease (PAD) and provide further insights into the underlying mechanism of PAD, based on a weighted gene co-expression network analysis (WGCNA) method. The mRNA expression profiles (accession. no. GSE27034), which included 19 samples from patients with PAD and 18 samples from normal control individuals were extracted from the Gene Expression Omnibus database. Subsequently, the differentially expressed genes (DEGs) were obtained using the Limma package and the co-expression network modules were screened using the WGCNA approach. In addition, the protein-protein interaction network for the DEGs in the most significant module was constructed using Cytoscape software. Functional enrichment analyses of the DEGs in the most significant module were also performed using the Database for Annotation, Visualization and Integrated Discovery and Kyoto Encyclopedia of Genes and Genomes (KEGG) Orthology-Based Annotation System, respectively. A total of 148 DEGs were identified in PAD, which were used to construct the WGCN, in which two modules (gray module and turquoise module) were identified, with the gray module exhibiting a higher gene significance (GS) value than the turquoise module. In addition, a co-expression network was constructed for $60 \mathrm{DEGs}$ in the gray module. The functional enrichment results showed that the DEGs in the gray module were enriched in five Gene Ontology terms and
\end{abstract}

Correspondence to: Dr Hao-Min Zhao or Dr Da-Jun Sun, Department of Vascular Surgery, China-Japan Union Hospital of Jilin University, 126 Xiantai Street, Changchun, Jilin 130033, P.R. China

E-mail: haominzhaoo@163.com

E-mail: dajunsund@163.com

Key words: differentially expressed genes, module, peripheral arterial disease, weighted gene co-expression network analysis four KEGG pathways. For example, cyclin-dependent kinase inhibitor 1A (CDKN1A), FBJ murine osteosarcoma viral oncogene homolog $(F O S)$ and prostaglandin-endoperoxide synthase 2 (PTGS2) were enriched in response to glucocorticoid stimulus. The results of the present study suggested that DEGs in the gray module, including CDKN1A, FOS and $P T G S 2$, may be associated with the pathogenesis of PAD, by modulating the cell cycle, and may offer potential for use as candidate treatment targets for PAD.

\section{Introduction}

Peripheral arterial disease (PAD), also known as peripheral vascular disease, is involved in the atherosclerotic occlusion of the arterial circulation to the lower extremities. The disease may manifest symptoms of intermittent claudication or severe chronic leg ischemia (1). The major risk factors for PAD are older age ( $>40$ years), cigarette smoking and diabetes mellitus (2). Patients diagnosed with PAD suffer from a limitation in exercise capacity and reduced quality of life, and are at increased risk of cardiovascular-associated morbidity and mortality $(3,4)$.

Due to poor prognosis and the increasing number of patients with PAD, an increasing number of investigations are focussed on the identifications of biomarkers for PAD, and aim to uncover the underlying mechanism of PAD. $\beta 2$-microglobulin has been considered to be a biomarker for PAD (5). In addition, Smadja et al found that thrombospondin 1 is significantly up-regulated in patients with PAD and may act as a plasmatic marker for PAD (6). Busti et al also reported that the plasma levels of certain matrix metalloproteinases (MMPs), including MMP-2 and MMP-9, are correlated with the development and severity of PAD development and severity (7). Previously, Masud et al screened 87 differentially expressed genes (DEGs) in PAD, and examined the functions and pathways of the DEGs by performing gene expression analysis of peripheral blood mononuclear cells (PBMCs) in patients with PAD and normal controls (8). However, the interactions among these genes and the exact molecular mechanism underlying PAD remain to be elucidated. 
Gene co-expression network-based approaches have been widely used in analyzing microarray data, particularly for the screening of functional modules $(9,10)$. One of the most useful gene co-expression network-based approaches is Weighted Gene Co-expression Network Analysis (WGCNA), which has been used to identify significant modules in a network and to screen candidate targets or biomarkers for human diseases or cancer (11-13). In the present study, the mRNA expression profiles were analyzed in peripheral blood mononuclear cell (PBMC) samples in patients diagnosed with PAD and in normal controls, to identify the DEGs associated with PAD. In addition, the significant modules were screened and a co-expression network was constructed using WGCNA. The distinct Gene Ontology (GO) functions and Kyoto Encyclopedia of Genes and Genomes (KEGG) pathways were also obtained to further examine the associations between the genes and PAD. These investigations aimed to provide evidence to further elucidated the mechanism underlying PAD.

\section{Materials and methods}

Gene expression datasets. The microarray expression data (accession. no. GSE27034) for PBMC samples in patients with PAD and controls without PAD (8) were obtained from the Gene Expression Omnibus (http://www.ncbi.nlm.nih.gov/ geo/) database (14). Gene expression analysis was performed based on a platform of [HG-U133_Plus_2] Affymetrix Human Genome U133 Plus 2.0 Array (GPL570; Affymetrix, Inc., Santa Clara, CA, USA). The datasets contained 37 samples, which included 19 samples from patients with PAD and 18 samples from normal controls.

Data preprocessing and screening of DEGs. The raw data were preprocessed via background correction, quantile normalization and probe summarization using the Affy package in R (Affymetrix, Inc.) (15). Subsequently, the expression profiling probes were transformed to corresponding gene symbols. In cases where there were more than one probe set in a single gene, the average expression values of all probes for the specific gene were defined as the gene expression value. In addition, when several mRNAs were mapped by one probe, this probe was considered to lack specificity and was excluded. Finally, the linear models for microarray data (Limma) package in R language (version 3.22.7; http://www. bioconductor.org/packages/3.0/bioc/html/limma.html) (16) was used to normalize the expression profiles and identify the DEGs between the PAD and normal samples. The genes identified with the cutoff criteria of fold change (FC) $>1.5$ and $\mathrm{P}$-value $<0.05$ were reserved as DEGs for subsequent analysis.

WGCNA. Initially, Pearson's correlation matrices for all gene pairs were calculated using SPSS software, version 16.0 (SPSS, Inc., Tokyo, Japan), and the correlation between gene $m$ and gene $n$ was defined as $\mathrm{S}_{\mathrm{mn}}=|\operatorname{cor}(\mathrm{m}, \mathrm{n})|$. Subsequently, the Pearson's correlation matrices were transformed into matrices of connection strengths using a power function, as follows $\mathrm{a}_{\mathrm{mn}}=$ power $\left(\mathrm{S}_{\mathrm{mn}}, \beta\right)=\left|\mathrm{S}_{\mathrm{mn}}\right|^{\beta}$. The $\beta$ value is set as the weighting coefficient only when the correlation coefficient between $\log (\mathrm{k})$ and $\log [\mathrm{p}(\mathrm{k})]$ reaches 0.9 , where $\mathrm{p}(\mathrm{k})$ represents the proportion of nodes with connectivity $(k)$. Thus, the $\beta$ value ( $\beta=16)$ was determined, so that the initial correlation coefficient reached 0.9 . Following determination of the adjacency parameter, the correlation matrix was transformed into an adjacency matrix, which was subsequently transformed into a topological overlap matrix (TOM). The TOM (17) was computed using the following formula:

$$
\varpi_{\mathrm{m} n}=\frac{l_{m n}+\alpha_{m n}}{\min \left\{k_{m}, k_{n}\right\}+1-\alpha_{m n}}
$$

where $l_{m n}$ indicates the sum of the products of the adjacency coefficients of the nodes connected to $m$ and $n . k_{m}$ indicates the sum of the adjacency coefficients of the nodes only connected to $m . k_{n}$ indicates the sum of the adjacency coefficient of the nodes only connected to $n$. If two nodes are not connected to each other and share no neighbors, $=0$.

Identification of significant modules and construction of the co-expression network. The obtained DEGs were hierarchically clustered using the dissimilarity coefficient as the distance measure, with each branch corresponding to a module. The modules containing at least 30 genes were assigned using the software of dynamic tree cut $\mathrm{R}$ package (version 1.62; Bioconductor, Los Angeles, CA, USA), which applies a novel dynamic branch cutting method for the detection of clusters in a dendrogram according to their shape (18). Following module detection, the eigengenes for each module were calculated, and the merged close modules were clustered into new modules. The module, which exhibited the maximal absolute correlation coefficient was considered to be the module that is most significantly associated with PAD. The network significance approach determines the module associated with PAD based on module significance (MS) (19). The MS indicates the average gene significance (GS) of all the genes in a module, and the GS of a gene is defined as - $\lg \mathrm{p}$, where $\mathrm{p}$ indicates the P-values obtained with Student's t-test using SPSS software, version 16.0. High MS values indicate a high association with PAD. In addition, the gene pairs, which were identified with the correlation coefficient $>0.7$ in the selected significant module were used to construct the co-expression network using Cytoscape software (version: 3.2.0; http://www.cytoscape.org/) (20).

Functional enrichment analyses. The Database for Annotation, Visualization and Integrated Discovery (DAVID) (21) was used to identify the enriched functions in the DEGs, with the cutoff criterion of a false discovery rate (FDR) $<0.05$. In addition, the KEGG Orthology-Based Annotation System (KOBAS) was applied to identify the significantly enriched pathways using a hypergeometric test (22) with the cutoff criterion of $\mathrm{P}<0.05$.

\section{Results}

Screening of DEGs. Under the cutoff criteria of FC $>1.5$ and $\mathrm{P}<0.05$, a total of 148 DEGs were obtained, which included 91 upregulated and 57 downregulated genes.

WGCNA analysis and identification of key modules. The 148 genes were subjected to WGCNA analysis, where the genes exhibiting similar patterns of expression were grouped into modules via hierarchical average linkage clustering (Fig. 1). The present study used two parameters (MS and P-value) to measure 
Table I. Enriched GO terms for the differentially expressed genes in the gray module.

\begin{tabular}{|c|c|c|c|c|c|}
\hline Accession no. & Term & Count & P-value & FDR & Gene \\
\hline GO: 0051384 & $\begin{array}{l}\text { Response to } \\
\text { glucocorticoid stimulus }\end{array}$ & 6 & $1.29 \mathrm{E}-05$ & 0.020401798 & $\begin{array}{l}C D K N 1 A, F O S, P T G S 2, D U S P 1, \\
A D M, A B C B 4\end{array}$ \\
\hline GO: 0010035 & $\begin{array}{l}\text { Response to } \\
\text { inorganic substance }\end{array}$ & 8 & $1.41 \mathrm{E}-05$ & 0.022301137 & $\begin{array}{l}P T P R K, F O S, C D K N 1 A, P T G S 2, \\
D U S P 1, N R 4 A 2, C A 2, T P M 1\end{array}$ \\
\hline GO: 0010033 & $\begin{array}{l}\text { response to } \\
\text { organic substance }\end{array}$ & 13 & $1.70 \mathrm{E}-05$ & 0.026762768 & $\begin{array}{l}\text { PTGS2, NR4A2, TLR4, GNG11, } \\
\text { PMAIP1, ABCB4,FOS, CDKN1A, } \\
\text { ACSL1, ADM, DUSP1, ID1, CA2 }\end{array}$ \\
\hline GO: 0031960 & $\begin{array}{l}\text { Response to } \\
\text { corticosteroid stimulus }\end{array}$ & 6 & $1.97 \mathrm{E}-05$ & 0.031048526 & $\begin{array}{l}F O S, C D K N 1 A, P T G S 2, D U S P 1 \\
A D M, A B C B 4\end{array}$ \\
\hline GO: 0009991 & $\begin{array}{l}\text { Response to } \\
\text { extracellular stimulus }\end{array}$ & 8 & $2.23 \mathrm{E}-05$ & 0.035204556 & $\begin{array}{l}F O S, C D K N 1 A, A C S L 1, P T G S 2, \\
D U S P 1, A D M, N R 4 A 2, K L F 4\end{array}$ \\
\hline
\end{tabular}

GO, gene ontology; FDR, false discovery rate.

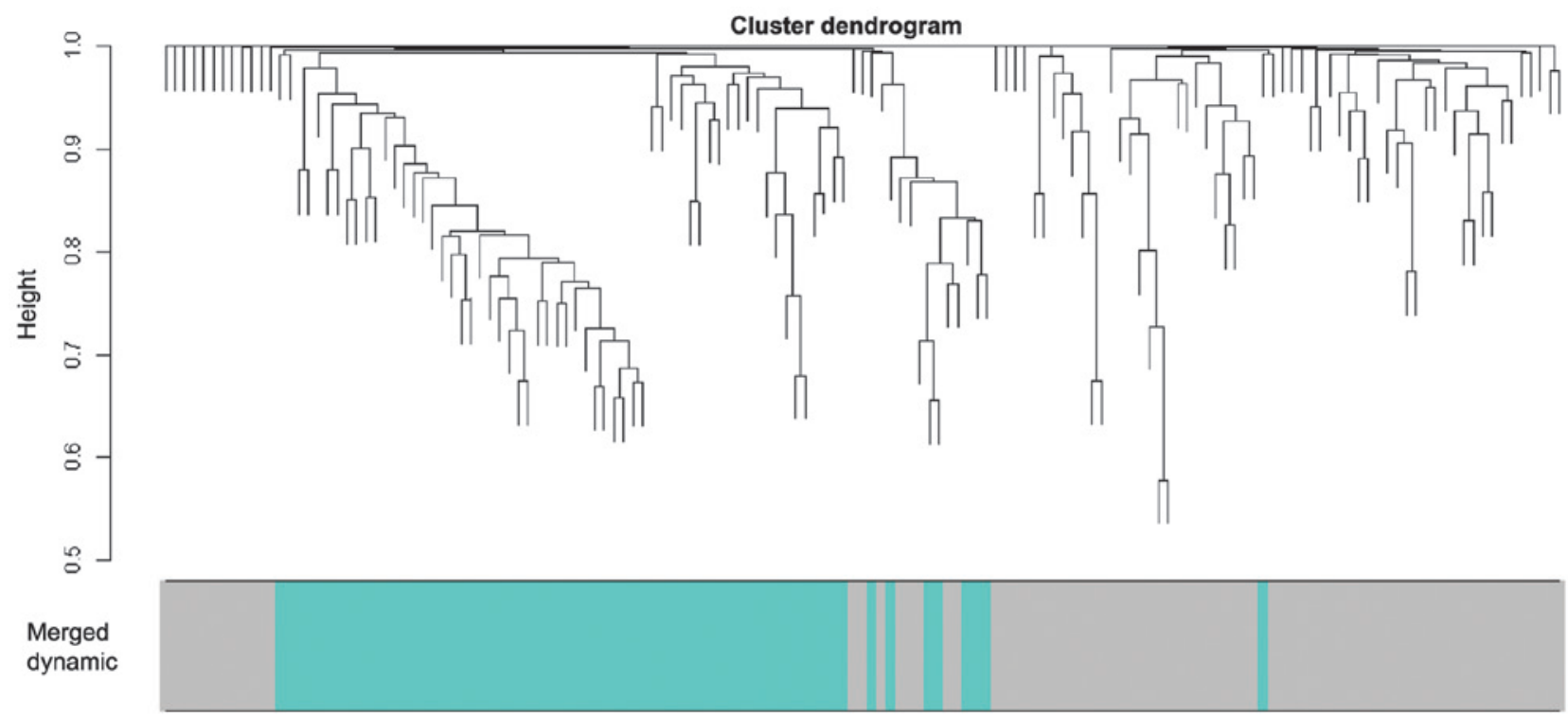

Figure 1. Cluster dendrogram and color display of the co-expression network modules. Each branch indicates a single gene. Each color indicates a single module. Height indicates the Euclidean distance. The area occupied by each color indicates the number of genes within the respective module.

the relevance between the modules and PAD. Initially, the MS value of each module was calculated, and the higher the MS value, the more relevant the correlation between the module and PAD was. In total, two modules (Fig. 2) were identified with a threshold of $\mathrm{P}<0.05$, which were termed the gray and turquoise modules. Furthermore, the gray module was found to exhibit a higher GS value, compared with the turquoise module, which suggested that the gray module was most significant. Finally, a co-expression network was constructed for the DEGs in the gray module with the cutoff criterion of a correlation coefficient $>0.7$ between two genes (Fig. 3). The resulting co-expression network contained a total of 60 genes and 167 interactions.

Functional enrichment analysis for DEGs in the gray module. Functional annotations and pathway enrichment analysis of the DEGs in gray module were performed on the basis of

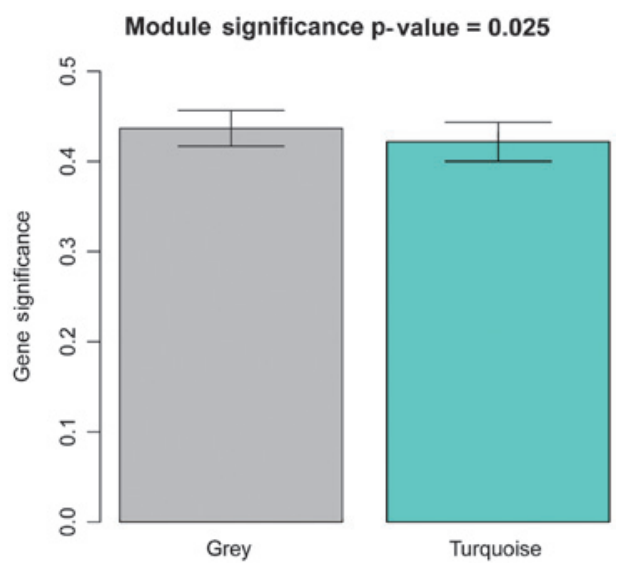

Figure 2. Significance of the co-expression network modules. The horizontal axis indicates the module name and the vertical axis indicates the value of gene significance. Gene significance $=\lg \mathrm{p}$, where $\mathrm{p}$ represents P-values. 
Table II. Enriched Kyoto Encyclopedia of Genes and Genomes database pathways for the differentially expressed genes in the gray module.

\begin{tabular}{|c|c|c|c|}
\hline Term & Description & Input & Gene \\
\hline hsa04012 & ErbB signaling pathway & $1839|1026| 2069$ & $H B E G F, C D K N 1 A, E R E G$ \\
\hline hsa04921 & Oxytocin signaling pathway & $2353|1026| 5743 \mid 2983$ & FOS, CDKN1A, PTGS2, GUCY1B3 \\
\hline hsa04713 & Circadian entrainment & $2983|2353| 2791$ & GUCY1B3, FOS, GNG11 \\
\hline hsa04620 & Toll-like receptor signaling pathway & 23531637317099 & FOS, CXCL11, TLR4 \\
\hline
\end{tabular}

Input, corresponding entrez gene ID for the genes.

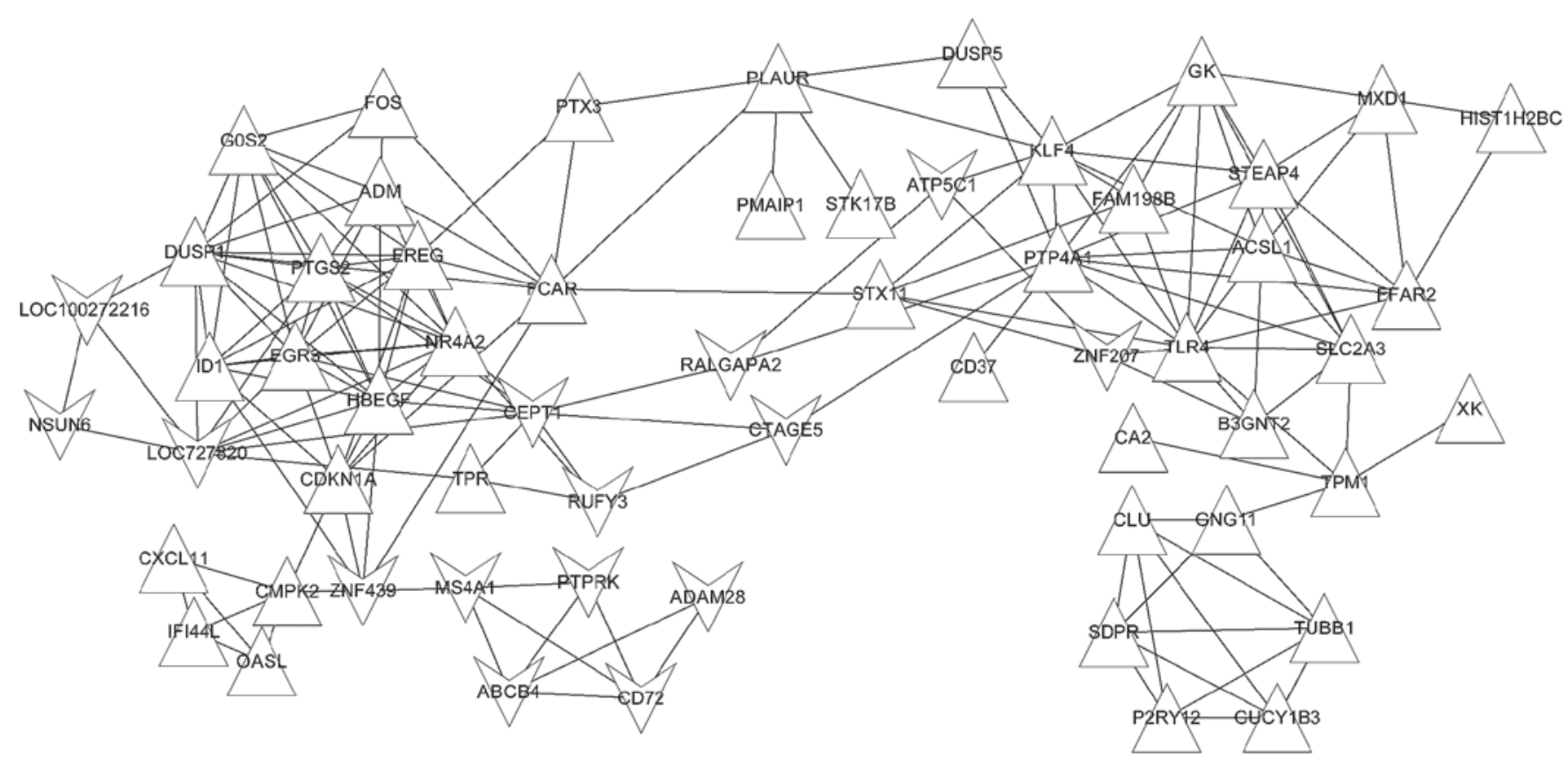

Figure 3. Co-expression network for the DEGs in gray module. The triangles indicate upregulated DEGs and the downward pointing arrows indicate downregulated DEGs. The lines represent interactions between genes. DEGs, differentially expressed genes.

their gene composition. The GO functional analysis revealed that the gray module was involved in responses to stimuli (Table I). For example, six DEGs, including cyclin-dependent kinase inhibitor 1A (CDKN1A), FBJ murine osteosarcoma viral oncogene homolog $(F O S)$ and prostaglandin-endoperoxide synthase 2 (PTGS2), were enriched in response to glucocorticoid stimulus; and eight DEGs, including PTPRK, FOS and CDKN1A, were enriched in response to inorganic substance. In addition, the pathway analysis indicated that the gray module was significantly involved in four pathways, including the ErbB signaling pathway (HBEGF, CDKN1A and EREG), oxytocin signaling pathway (FOS, CDKNIA and PTGS2), circadian entrainment (GUCY1B3, FOS, GNG11) and Toll-like receptor signaling pathway (FOS, CXCL11, TLR4), as presented in Table II.

\section{Discussion}

PAD is among the common manifestations of systemic atherosclerosis, which affects other major circulations involving the cerebral and coronary circulations (23). However, the molecular mechanism underlying the disease remains to be fully elucidated. In the present study, two significant modules involved in PAD were identified and screened, and the co-expressed network was constructed using the WGCNA method by bioinformatics analysis. The present study found that certain genes in the most significant module were closely associated with the response to glucocorticoid stimulus, determined using enrichment analysis. The present study aimed to screen the important modules and genes, which were identified to be associated with PAD and examine the molecular mechanism of PAD.

The two modules were obtained using WGCNA, of which the gray module was found to be the most significant module. The genes in the gray module were associated with the responses to stimulus. CDKN1A, FOS and PTGS2 were significantly upregulated in the gray module. CDKN1A, also known as p21/WAF1/CIP1, is a regulator of cell cycle progression at the $G_{1}$ and $S$ phase (24), which is required for the response to DNA damage by inducing cell cycle arrest, inhibiting DNA replication and regulating fundamental cellular processes, including apoptosis and gene 
transcription (25). Furthermore, $C D K N 1 A$ can interaction with proliferating cell nuclear antigen, which is a DNA polymerase accessory factor and has a regulatory role in DNA damage repair and the $S$ phase of DNA replication $(25,26)$. In addition, $C D K N 1 A$ has also been found to be associated with atherosclerosis and myocardial infarction (27). The results of the present study also showed that $C D K N 1 A$ was involved in the responses to stimulus, including the response to glucocorticoid stimulus, response to inorganic substance and response to organic substance. Therefore, the present study suggested that $C D K N 1 A$ may be involved in the processes and pathways, which are associated with the cell cycle in patients with PAD.

FOS/c-FOS is a proto-oncogene, which is induced by a variety of extracellular stimuli (28). FOS acts as a transcription factor, which promotes the expression of specific cell cycle regulatory genes (29) and leads to early G1-phase cyclin accumulation, and enhances cyclin D- and cyclin E-associated kinase activities (30). In addition, it has been reported that FOS interacts with the c-jun proto-oncogene to form transcription factor activating protein 1 (31), which is a crucial protein required for cell adaptation to environmental changes (32). PTGS2, also termed cyclooxygenase-2, is unexpressed under normal conditions in the majority of cells. The inhibition of PTGS2 has been shown to improve endothelial function in coronary artery disease (33). Additionally, PTGS2 has been found to interact with caveolin 1 (34), which is a predominant component of caveolae plasma membranes and is associated with the repair of DNA damage through regulating the important molecules involved in maintaining genomic integrity (35). Thus, FOS and PTGS2 are also cell cycle-associated genes and may be involved in the pathogensis of PAD.

In conclusion, the present study used the WGCNA approach to analyze the mRNA expression profile of PAD, and obtained two key modules. The results suggested that the gray module-associated genes may be associated with the development of PAD, particularly CDKN1A, FOS and $P T G S 2$. These genes may be involved in the pathogenesis of PAD by regulating the cell cycle, and offer potential for use as potential therapeutic targets for PAD. However, further experiments are required to verify these results.

\section{References}

1. Hiatt WR and Nehler MR: Peripheral arterial disease. 2007.

2. Hiatt WR: Medical treatment of peripheral arterial disease and claudication. N Engl J Med 344: 1608-1621, 2001.

3. Cronberg C, Sjöberg S, Albrechtsson U, Leander P, Lindh M, Norgren L, Danielsson P, Sonesson B and Larsson EM: Peripheral arterial disease. Contrast-enhanced 3D MR angiography of the lower leg and foot compared with conventional angiography. Acta Radiol 44: 59-66, 2003.

4. Ouriel K: Peripheral arterial disease. Lancet 358: 1257-1264, 2001.

5. Wilson AM, Kimura E, Harada RK, Nair N, Narasimhan B, Meng XY, Zhang F, Beck KR, Olin JW, Fung ET and Cooke JP: Beta2-Microglobulin as a biomarker in peripheral arterial disease: Proteomic profiling and clinical studies. Circulation 116: 1396-1403, 2007.

6. Smadja DM, D'audigier C, Bièche I, Evrard S, Mauge L, Dias JV, Labreuche J, Laurendeau I, Marsac B, Dizier B, et al: Thrombospondin-1 is a plasmatic marker of peripheral arterial disease that modulates endothelial progenitor cell angiogenic properties. Arterioscler Thromb Vasc Biol 31: 551-559, 2011.
7. Busti C, Falcinelli E, Momi S and Gresele P: Matrix metalloproteinases and peripheral arterial disease. Intern Emerg Med 5: 13-25, 2010.

8. Masud R, Shameer K, Dhar A, Ding K and Kullo IJ: Gene expression profiling of peripheral blood mononuclear cells in the setting of peripheral arterial disease. J Clin Bioinforma 2: 6 , 2012.

9. Horvath S and Dong J: Geometric interpretation of gene coexpression network analysis. PLoS Comput Biol 4: e1000117, 2008.

10. Ruan J, Dean AK and Zhang W: A general co-expression network-based approach to gene expression analysis: Comparison and applications. BMC Syst Biol 4: 8, 2010.

11. Malki K, Tosto MG, Jumabhoy I, Lourdusamy A, Sluyter F, Craig I, Uher R, McGuffin P and Schalkwyk LC: Integrative mouse and human mRNA studies using WGCNA nominates novel candidate genes involved in the pathogenesis of major depressive disorder. Pharmacogenomics 14: 1979-1990, 2013.

12. Udyavar AR, Hoeksema MD, Clark JE, Zou Y, Tang Z, Li Z, Li M, Chen H, Statnikov A, Shyr Y, et al: Co-expression network analysis identifies spleen tyrosine kinase (SYK) as a candidate oncogenic driver in a subset of small-cell lung cancer. BMC Syst Biol 7 (Suppl 5): S1, 2013.

13. Zhao H, Cai W, Su S, Zhi D, Lu J and Liu S: Screening genes crucial for pediatric pilocytic astrocytoma using weighted gene coexpression network analysis combined with methylation data analysis. Cancer Gene Ther 21: 448-455, 2014.

14. Barrett T, Wilhite SE, Ledoux P, Evangelista C, Kim IF, Tomashevsky M, Marshall KA, Phillippy KH, Sherman PM, Holko M, et al: NCBI GEO: Archive for functional genomics data sets-update. Nucleic Acids Res 41: D991-D995; Database issue, 2013

15. Gautier L, Cope L, Bolstad BM and Irizarry RA: affy-analysis of affymetrix genechip data at the probe level. Bioinformatics 20: 307-315, 2004.

16. Smyth GK: Limma: Linear models for microarray data. In: Bioinformatics and Computational Biology Solutions Using $\mathrm{R}$ and Bioconductor. Gentleman R, Carey V, Huber W, Irizarry R and Dudoit S (eds). Springer, New York, pp397-420, 2005.

17. Yip AM and Horvath S: Gene network interconnectedness and the generalized topological overlap measure. BMC Bioinformatics 8: 22, 2007.

18. Langfelder P, Zhang B and Horvath S: Defining clusters from a hierarchical cluster tree: The dynamic tree cut package for $\mathrm{R}$. Bioinformatics 24: 719-720, 2008

19. Langfelder P and Horvath S: WGCNA: An R package for weighted correlation network analysis. BMC Bioinformatics 9: 559-571, 2008.

20. Shannon P, Markiel A, Ozier O, Baliga NS, Wang JT, Ramage D, Amin N, Schwikowski B and Ideker T: Cytoscape: A software environment for integrated models of biomolecular interaction networks. Genome Res 13: 2498-2504, 2003.

21. Huang Da W, Sherman BT, Tan Q, Collins JR, Alvord WG, Roayaei J, Stephens R, Baseler MW, Lane HC and Lempicki RA: The DAVID gene functional classification tool: A novel biological module-centric algorithm to functionally analyze large gene lists. Genome Biol 8: R183, 2007.

22. Wu J, Mao X, Cai T, Luo J and Wei L: KOBAS server: A web-based platform for automated annotation and pathway identification. Nucleic Acids Res 34: W720-W724, 2006.

23. American Disease Association: Peripheral arterial disease in people with diabetes. J Am Podiatr Med Assoc 95: 309-319, 2005.

24. Gartel AL and Radhakrishnan SK: Lost in transcription: p21 repression, mechanisms and consequences. Cancer Res 65: 3980-3985, 2005.

25. Cazzalini O, Scovassi AI, Savio M, Stivala LA and Prosperi E: Multiple roles of the cell cycle inhibitor p21(CDKN1A) in the DNA damage response. Mutat Res 704: 12-20, 2010.

26. Gulbis JM, Kelman Z, Hurwitz J, O'donnell M and Kuriyan J: Structure of the C-terminal region of p21(WAF1/CIP1) complexed with human PCNA. Cell 87: 297-306, 1996.

27. Rodriguez I, Coto E, Reguero JR, González P, Andrés V, Lozano I, Martín M, Alvarez V and Morís C: Role of the CDKN1A/p21, CDKN1C/p57 and CDKN2A/p16 genes in the risk of atherosclerosis and myocardial infarction. Cell Cycle 6: 620-625, 2007

28. Abate C, Luk D, Gagne E, Roeder RG and Curran T: Fos and jun cooperate in transcriptional regulation via heterologous activation domains. Mol Cell Biol 10: 5532-5535, 1990.

29. Hunter T: Oncoprotein networks. Cell 88: 333-346, 1997. 
30. Braun-Dullaeus RC, Mann MJ and Dzau VJ: Cell cycle progression New therapeutic target for vascular proliferative disease. Circulation 98: 82-89, 1998.

31. Mahner S, Baasch C, Schwarz J, Hein S, Wölber L, Jänicke F and Milde-Langosch K: C-Fos expression is a molecular predictor of progression and survival in epithelial ovarian carcinoma. $\mathrm{Br}$ J Cancer 99: 1269-1275, 2008.

32. Bossis G, Malnou CE, Farras R, Andermarcher E, Hipskind R, Rodriguez M, Schmidt D, Muller S, Jariel-Encontre I and Piechaczyk M: Down-regulation of c-Fos/c-Jun AP-1 dimer activity by sumoylation. Mol Cell Biol 25: 6964-6979, 2005.
33. Chenevard R, Hürlimann D, Béchir M, Enseleit F, Spieker L, Hermann M, Riesen W, Gay S, Gay RE, Neidhart M, et al: Selective COX-2 inhibition improves endothelial function in coronary artery disease. Circulation 107: 405-409, 2003.

34. Liou JY, Deng WG, Gilroy DW, Shyue SK and Wu KK Colocalization and interaction of cyclooxygenase-2 with caveolin-1 in human fibroblasts. J Biol Chem 276: 34975-34982, 2001.

35. Zhu H, Yue J, Pan Z, Wu H, Cheng Y, Lu H, Ren X, Yao M, Shen Z and Yang JM: Involvement of Caveolin-1 in repair of DNA damage through both homologous recombination and non-homologous end joining. PloS One 5: e12055, 2010. 